\title{
Topological solitons, cholesteric fingers and singular defect lines in Janus liquid crystal shells
}

${ }_{++}^{+}$Received 00th January 20xx,
Accepted 00th January 20xx

DOI: $10.1039 / \mathrm{x} 0 \times x \times 00000 \mathrm{x}$

\author{
Guillaume Durey, ${ }^{\mathrm{a}}$ Hayley R. O. Sohn, ${ }^{\mathrm{b}}$ Paul J. Ackerman, ${ }^{\mathrm{c}}$ Etienne Brasselet, ${ }^{\mathrm{d}}$ Ivan I. Smalyukh ${ }^{\mathrm{bce}}$ and \\ Teresa Lopez-Leon*a
}

\begin{abstract}
Topological solitons are non-singular but topologically nontrivial structures in fields, which have fundamental significance across various areas of physics, similar to singular defects. Production and observation of singular and solitonic topological structures remain a complex undertaking in most branches of science - but in soft matter physics, they can be realized within the director field of a liquid crystal. Additionally, it has been shown that confining liquid crystals to spherical shells using microfluidics resulted in a versatile experimental platform for the dynamical study of topological tranformations between director configurations. In this work, we demonstrate the triggered formation of topological solitons, cholesteric fingers, singular defect lines and related structures in liquid crystal shells. We show that to accommodatethese objects, shells must possess a Janus nature, featuring both twisted and untwisted domains. We report the formation of linear and axisymmetric objects, which we identify as cholesteric fingers and skyrmions or elementary torons, respectively. We then take advantage of the sensitivity of shells to numerous external stimuli to induce dynamical transitions between various types of structures, allowing for a richer phenomenology than traditional liquid crystal cells with solid flat walls. Using gradually more refined experimental techniques, we induce the targeted transformation of cholesteric twist walls and fingers into skyrmions and elementary torons. We capture the different stages of these director transformations using numerical simulations. Finally, we uncover an experimental mechanism to nucleate arrays of axisymmetric structures on shells, thereby creating a system of potential interest for tackling crystallography studies on curved spaces.
\end{abstract}

\section{Introduction}

Topological solitons - spatially localized, non-singular configurations in a vector field that cannot be transformed continuously into a uniform state - are a prime example of a historically theoretical phenomenon. ${ }^{1}$ Similar to their singular defect counterparts, they have significance in condensed matter, particle, nuclear, and atomic physics, as well as in cosmology. ${ }^{1}$ Liquid crystals are a rich model system for experimentally studying phenomena in classical field theory that are typically restricted to a pure mathematical treatment. ${ }^{2,3}$ Cholesteric liquid crystals display a spontaneous tendency to form twisted configurations of the director, the field which represents the average mesoscopic orientation of the liquid crystal molecules. This tendency preferentially leads to the formation of uniformly twisted states but can also stabilize topological solitons. ${ }^{4-7}$ Indeed, perpendicular

\footnotetext{
a. Laboratoire Gulliver, UMR CNRS 7083, ESPCI Paris, Université PSL, 10 rue Vauquelin, 75005 Paris, France. Email: teresa.lopez-leon@espci.fr.

b. Soft Materials Research Center and Materials Science and Engineering Program, University of Colorado, Boulder, Colorado 80309, USA.

c. Department of Physics and Department of Electrical, Computer and Energy Engineering, University of Colorado, Boulder, Colorado 80309, USA.

d. Univ. Bordeaux, CNRS, LOMA, UMR 5798, F-33400 Talence, France.

e. Renewable and Sustainable Energy Institute, National Renewable Energy Laboratory and University of Colorado, Boulder, Colorado 80309, USA.

Electronic Supplementary Information (ESI) available: [details of any supplementary information available should be included here]. See DOI: 10.1039/x0xx00000x
}

boundary conditions, also referred to as homeotropic anchoring, are incompatible with uniform twist. Under such frustrated conditions, it is often energetically favorable for highly confined cholesteric samples to untwist entirely, resembling homeotropically-aligned uniform nematics. Slightly less confined cholesterics relieve their geometrical frustration by forming structures of localized twist embedded in unwound domains, which can arise either spontaneously or with the assistance of external stimuli. ${ }^{8-18}$ Two broad classes of twisted, spatially-localized objects are known to form: linear structures called cholesteric fingers, and axially symmetric particle-like configurations. Among the latter, skyrmionic structures have generated the most interest. The simplest type of skyrmions are field configurations with circular symmetry, in which the field twists in all directions from the center outwards through an angle $\pi$. As they could become the building blocks of prospective paradigm-shifting memory storage devices, skyrmions have attracted a lot of attention in magnetism over the last few years. ${ }^{19-22}$

Historically, various localized twisted cholesteric structures ${ }^{8-18}$ were first reported in wedge systems, where a cholesteric is sandwiched between plates forming a small angle - which enables a static, linear spatial variation in confinement - or in flat cells with parallel walls. As the director configuration and topology were initially uncharacterized, all particle-like excitations were originally labelled with generic names, such as "cholesteric bubbles" or "spherulites". Since 
then, progress in three-dimensional imaging techniques and numerical simulations have spurred a large number of studies, that revealed the precise director configurations of both linear fingers ${ }^{4}$ and axisymmetric structures. ${ }^{5-7}$ The latter have been shown to possess a wide topological diversity, which is not always perceptible when only using polarized microscopy. ${ }^{5-7}$

Meanwhile, a different geometry has been shown to constitute a versatile platform for the dynamical study of liquid crystalline structures: liquid crystal shells, double emulsions where the liquid crystal is confined to a spherical film between two water phases. Shells have recently attracted a lot of attention owing to their unique properties. ${ }^{23,24}$ Under planar anchoring, i.e. when the boundary conditions force the molecules to lie parallel to the interfaces, their spherical shape stabilizes singularities in the director field ${ }^{25}-$ a topological requirement stated by the Poincaré-Hopf theorem. ${ }^{26,27}$ Their all-fluid nature enables both easy access to their boundaries and facile control of their thickness. Shells constitute an ideal system for answering fundamental questions on the nature of liquid crystal mesophases. In particular, previous works on cholesteric shells have shed light on the constitution and the dynamics of defects under planar anchoring, ${ }^{28,29}$ and on the formation of patterns under homeotropic anchoring with moderate strength. ${ }^{30}$ They are also particularly suitable for applications in biochemical sensing ${ }^{31,32}$ or photonics. ${ }^{33-35}$ In short, shells support complex liquid crystal structures and are an efficient and precise way to investigate the dynamics of transformations between them.

In this article, we uncover the phenomenology of cholesteric shells under strong homeotropic anchoring and strong confinement of the liquid crystal. In section 3.1, we report their ability to spontaneously adopt a Janus nature, displaying a coexistence of twisted and untwisted domains, and to stabilize a variety of structures of localized twist including cholesteric fingers and elementary torons. We combine the various experimental and numerical tools developed throughout a decade to shine light on the nature of these structures. In section 3.2, we trigger dynamical transitions between these objects. We show that these transformations are qualitatively richer than those performed with traditional flat and wedge-shaped cells. And lastly, we focus on the structural transformations that result in the formation of axisymmetric solitons. We explore a variety of experimental strategies to produce such structures in the curved space of a shell.

\section{Experimental and numerical}

\subsection{Shell preparation}

We used 4'-pentyl-4-cyanobiphenyl (5CB) doped with (S)-4-cyano-4'(2-methylbutyl)biphenyl (CB15) as our cholesteric liquid crystal. The amount of dopant determines the cholesteric pitch, $p$, i.e. the distance required for the director to twist by a full turn. ${ }^{36}$ Unless otherwise stated, we used a $3 \%$ wt mixture, yielding a $4.7 \mu \mathrm{m}$ pitch. We produced cholesteric shells using traditional capillary microfluidic devices, ${ }^{37}$ in which a liquid crystal jet envelops a smaller jet of inner solution, forming a compound jet that is sheared by an outer solution and breaks up into spherical shells, having a typical outer diameter of $50 \mu \mathrm{m}$ to $100 \mu \mathrm{m}$. Both the inner and outer solutions were waterbased, and contained $1 \%$ wt polyvinyl alcohol (PVA, $13-23 \mathrm{~kg} / \mathrm{mol}$ ), a polymer surfactant that enhances the stability of the double emulsions. Additionally, the outer solution contained $60 \% \mathrm{wt}$ glycerol, which helps increase the viscosity of the outer phase, giving more control over the size of the shells during their production. Depending on the experiments, other solutes were added to the inner phase: namely, sodium dodecyl sulfate (SDS) and/or sodium chloride ( $\mathrm{NaCl}$ ). SDS enforces homeotropic anchoring at the water / liquid crystal interface. $\mathrm{NaCl}$ is mainly used as an osmolyte, to induce shell swelling, but it also screens the electrostatic repulsions between the surfactants, inducing a denser packing on the interfaces. ${ }^{38}$ All chemicals were purchased from Sigma-Aldrich, except for the two liquid crystals, which were bought from Synthon Chemicals, and except for the phototunable dopant OA-1 (9-(2-phenyl-2,3-dihydro-cyclopenta[a]naphthalen-1-ylidene)-9H-fluorene) used in section 3.2.2, which was synthesized at AlphaMicron, Inc. ${ }^{39-42}$ We used deionized water produced by a Millipore Synergy fountain.

\subsection{Shell observation}

The outlet of the microfluidic device was connected to a vial filled with a water-based collection solution, also containing $1 \%$ wt PVA and additional solutes depending on the experiment. Unless otherwise stated, the inner and collection solutions were both $1 \%$ wt PVA, $20 \mathrm{mM}$ SDS in water, thus enforcing strong perpendicular anchoring at both interfaces of the shells. The contents of the vial were carefully homogenized once enough shells had been collected. $200 \mu \mathrm{m}$ thick observation chambers were built by stacking two layers of doublesided tape (Gudy 804, Filmolux) with a circular hole on a glass slide. Shells were then pipetted from the vial to an observation chamber, which was sealed with a coverslip. Unless otherwise stated, the middle phase was slightly denser than the inner and collection phases in our experiments. Therefore, shells sedimented on the bottom slide of the chamber, and were thickest at their South pole (between $1 \mu \mathrm{m}$ and $25 \mu \mathrm{m}$ ) and thinnest at their North pole (less than $1 \mu \mathrm{m}$ ), the poles of the shells being defined with respect to the vertical direction. Shells were imaged with Nikon DSLR cameras (D5300 or D300s), mounted on an upright Nikon $\mathrm{Ni}-\mathrm{U}$ polarizing microscope, equipped with an Instec TS62 thermal stage, driven by a mK1000 unit. For electric field experiments, the cover slip and the glass slide of the observation chamber were replaced by two ITO-coated glass slides (12-15 $\Omega / s q$, VisionTek Systems Ltd.), cleaned with deionized water, ethanol and acetone. In both slides, the coating was in contact with the continuous water phase and glued to a conducting wire. A waveform generator (Agilent Technologies 33210A) was used to impose a voltage between the two wires, visualized with an oscilloscope (Agilent Technologies DSO-X 2002A).

\subsection{Shell manipulation}

For osmotic swelling experiments, we used a 1\% wt PVA, 10 mM SDS, $1.1 \mathrm{M} \mathrm{NaCl}$ water solution as the inner water phase, and a 1\% wt PVA, $10 \mathrm{mM} \mathrm{SDS}, 0.1 \mathrm{M} \mathrm{NaCl}$ solution as the outer phase. For field unwinding experiments, we used $20 \mathrm{mM}$ SDS, $1 \%$ wt PVA water solutions as the inner and collection phases. There were also traces of glycerol in the collection phase, coming from the outer phase used in the microfluidic device. Shells were pipetted from the collection vial and sedimented to the bottom of the observation chamber, resting on the 
ITO coating of the bottom slide. As the observation chamber is essentially water, with a significant concentration of ionic species, mostly coming from SDS and its sodium counter-ions, it is not a perfect insulator. Using a constant voltage induces a migration of the ions in solution towards the oppositely charged electrode, screening the electric field in the bulk of the chamber. It also induces a chemical reaction at the cathode rendering its ITO coating opaque. Therefore, we made sure that the direct component of the voltage we applied was strictly set to zero. We found the resistance of our observation chambers to be both frequency-dependent and sample-dependent. We found that the optimal results were obtained with either a $1 \mathrm{kHz}$ or a $1 \mathrm{MHz}$ frequency, for $10 \mathrm{~V}$ peak-to-peak alternating square waves. At these frequencies, the actual voltage measured across the observation chamber was up to half the voltage imposed by the function generator. Electric fields were applied in short periods, a few minutes at most, and all periods combined did not exceed ten minutes for each sample. Samples were not re-used.

\subsection{Computer simulations}

The simulations of chiral nematic structures in spherical shell geometries were performed using a numeric

al technique based on iterative minimization of the Frank-Oseen elastic free energy of the bulk material, $F_{\text {bulk }}^{\mathrm{FO}}$, where $K_{1}, K_{2}$, and $K_{3}$ are the elastic constants for splay, twist, and bend deformation costs of the director field, $\boldsymbol{n}(\boldsymbol{r}):$ 5,7,43

$$
\begin{aligned}
F_{\mathrm{bulk}}^{\mathrm{FO}}=\int & \left\{\frac{K_{1}}{2}(\nabla \cdot \boldsymbol{n})^{2}+\frac{K_{2}}{2}[\boldsymbol{n} \cdot(\nabla \times \boldsymbol{n})+q]^{2}\right. \\
& \left.+\frac{K_{3}}{2}[\boldsymbol{n} \times(\nabla \times \boldsymbol{n})]^{2}\right\} d^{3} \boldsymbol{r}
\end{aligned}
$$

The wavenumber of the ground-state chiral nematic mixture is defined as $q=2 \pi / p$. The ground-state helicoidal pitch, $p$, is determined by the mass fraction of the chiral dopant, $C$, and the helical twisting power, $H_{0}$, according to the relation $p=1 /\left(H_{0} C\right)$. The material parameters for the numerical simulations are consistent with a 5CB-CB15 cholesteric mixture (Table 1), where $H_{0}=7.3 \mu \mathrm{m}^{-1}$. Whenever the surface anchoring energy is considered finite and taken into account, Eq. (1) is supplemented by the Rapini-Papoular surface anchoring potential ${ }^{44}$ :

$$
F_{\text {surface }}^{\mathrm{FO}}=-\int \frac{W}{2}\left(\boldsymbol{n} \cdot \boldsymbol{v}_{0}\right)^{2} d^{2} r
$$

Where $W$ is the surface anchoring strength and $\boldsymbol{v}_{0}$ is the easy axis orientation at the shell's surfaces. Because the experimental measurement of the surface anchoring strength, $W$, is difficult in our experimental geometry under varying experimental conditions and is beyond the scope of this study, we also do not perform a systematic investigation of the role of $F_{\text {surface }}^{\mathrm{FO}}$ energetic contribution in our simulations. We therefore don't explore the anchoring effects on the relative stability of structures we discuss, although recent work ${ }^{45}$ shows the correlation between anchoring strength and three-dimensional structural details of topological solitons in flat cell geometry where The minimization of free energy was achieved by an iterative finite difference scheme using radial basis functions, an approach for simulation of liquid crystal director dynamics which has been described

Table 1. Material properties of nematic host 5CB. Parameters characterizing elastic $K_{1}, K_{2}, K_{3}$ constants and optical anisotropy, $\Delta n$.

\begin{tabular}{|l|l|l|l|c|}
\hline Material/Property & $K_{1}(\mathrm{pN})$ & $K_{2}(\mathrm{pN})$ & $K_{3}(\mathrm{pN})$ & $\Delta n$ \\
\hline $5 \mathrm{CB}$ & 6.4 & 3 & 10 & 0.18 \\
\hline
\end{tabular}

This journal is (C) The Royal Society of Chemistry 20xx
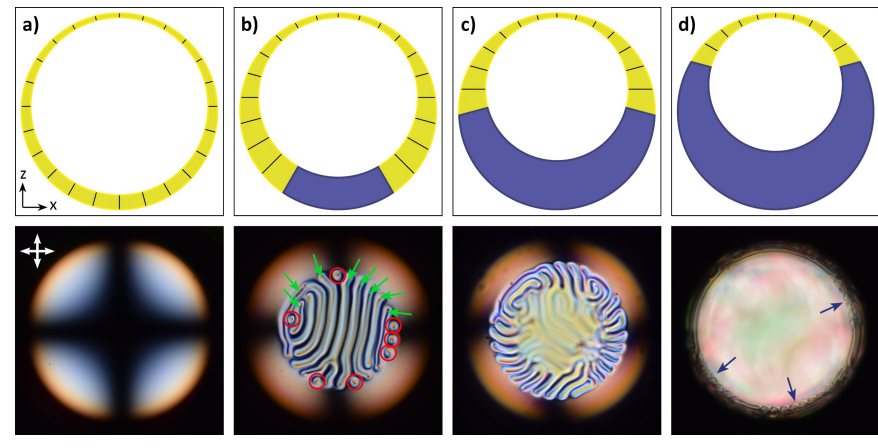

Fig. 1 Cholesteric shells with increasing mean thickness under strong perpendicular anchoring. Top: sketch of a side view of the geometry, showing twisted domains (purple), untwisted domains (yellow) and a radial director field in the untwisted domains (black lines). Bottom: polarized microphotographs showing a top view of the shells, which are all about $200 \mu \mathrm{m}$ in outer diameter. White double arrows denote crossed polarizer orientations. In b), axisymmetric structures are circled in red and each cholesteric finger is identified with an arrow (some fingers are branched). In d), dark blue arrows denote the location of small cholesteric fingers at the interface between twisted and untwisted domains.

previously. ${ }^{46}$ The spherical confining surfaces were defined by drawing two nested spherical Fibonacci grids ${ }^{47}$ to preserve the equal-area property of grid points. These nested grids were then used as the input boundaries for the generation of a tetrahedral mesh (TetGen, WIAS) representing the node locations within the thin spherical shell volume of liquid crystalline material. The director field boundary conditions were mimicking homeotropic alignment of $\boldsymbol{n}(\boldsymbol{r})$ with respect to the liquid crystal interface. Numerical modeling provided insights into the possible nature of some of the twisted structures seen in experiments, including cholesteric fingers, torons and skyrmions.

\section{Results and discussion}

\subsection{Janus liquid crystal shells: a zoo of topological structures}

A cholesteric liquid crystal shell whose interfaces are saturated with SDS molecules provides an analogous environment to the wedges in which fingers and spherulites were first reported, as it i) frustrates the cholesteric by imposing boundary conditions that are incompatible with its tendency to twist, and ii) features a monotonous spatial variation in the local cholesteric thickness. Indeed, on the one hand, amphiphilic molecules such as SDS locate preferentially at water / liquid crystal interfaces, forcing the neighboring liquid crystal molecules to align with their aliphatic tails, hence enforcing homeotropic anchoring. On the other hand, the water and liquid crystal phases always have a slightly different density: this creates a buoyancy force that typically lifts the inner water droplet up inside the shell, making the spherical liquid crystal membrane thinnest at the North pole of the shell, and thickest at its South pole.

Under these conditions, the liquid crystal textures on the shells are governed by one parameter: the ratio of the local thickness $h$ to the equilibrium cholesteric pitch $p$, known as the confinement ratio. ${ }^{48}$ Keeping the pitch constant, we produce shells with variable mean thicknesses, yielding a rich phenomenology depicted in Fig. 1 . When a shell is thin enough that for all latitudes $h / p<1$, one gets a standard radial nematic shell, shown in Fig. $1 \mathrm{a}$. It is easily recognizable due 

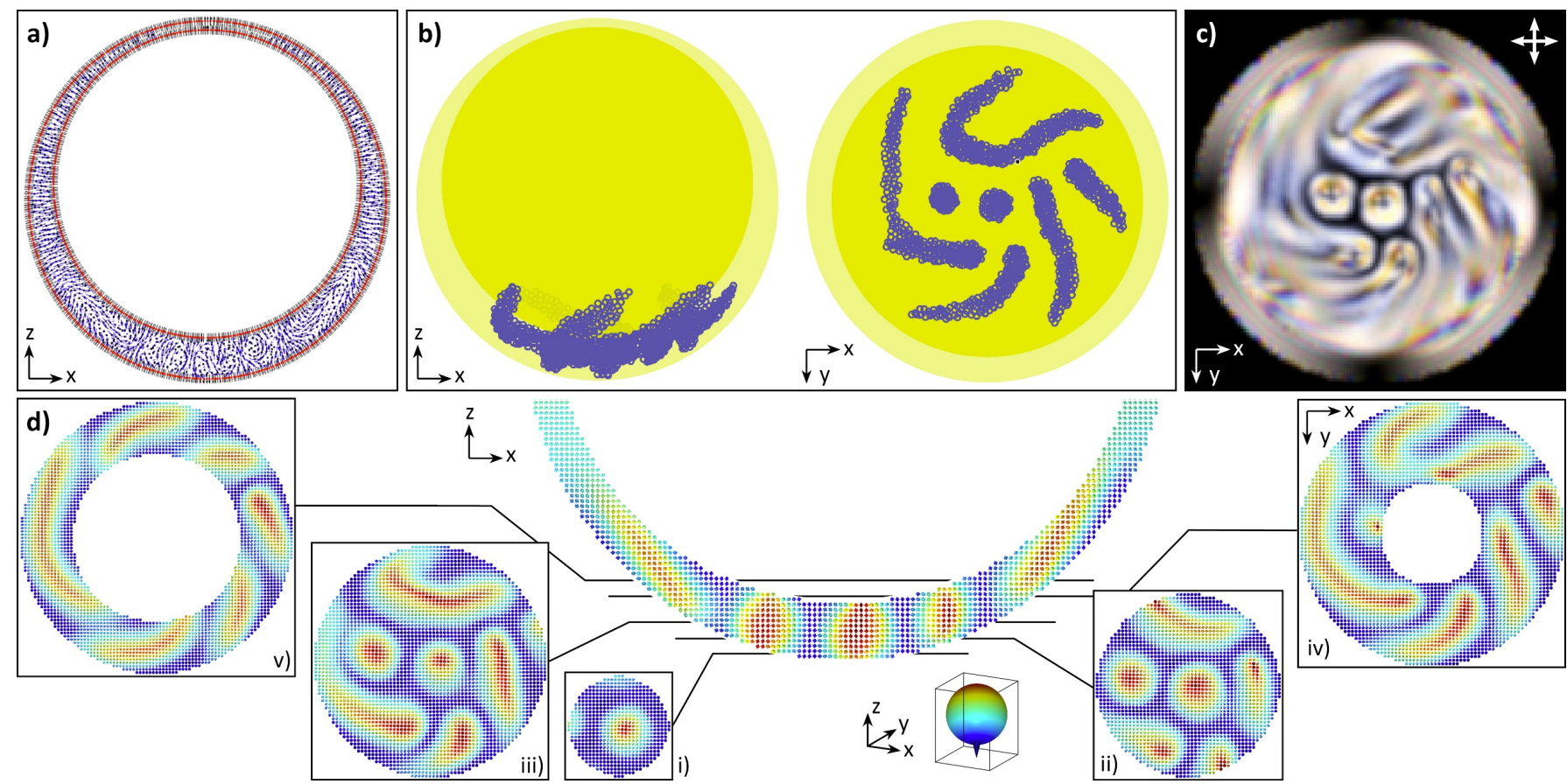

Fig. 2 Computer-simulated cholesteric structures on shells. a) Vertical 2D cross-section through a cholesteric shell having a local confinement ratio in the South pole of $h / p=1.25$, showing the director field (blue dashes) at every computational node (black dots), in the presence of strong homeotropic boundary conditions (black dashes at red dots). b) Side view (left) and top view (right) of the shell in 3D, showing the regions of high twist in the director field using purple markers, and the two shell interfaces as yellow spheres. c) Top view of the simulated polarized microscopy texture of the same shell, with an approximate diameter of $75 \mu \mathrm{m}$. White double arrows denote crossed polarizer orientations. d) Center: close-up on the bottom part of a vertical cross-section of the same shell, with a vectorized director field, revealing the inner structure of the topological solitons. Each color represents a different orientation of the vectorized director on the $\mathbb{S}^{2}$ unit sphere (inset) order parameter space. From i) to v): series of horizontal cross-sections at the locations indicated by black lines, at increasing altitudes. Computer simulations emphasize that liquid crystal shells of varying thickness embed cholesteric configurations in fundamentally more complex way than flat and wedge-shaped cells, allowing co-existence of elementary skyrmions and topologically trivial twisted structures filling in the space between spherical surfaces.

to the large Maltese cross pattern it displays under a polarizing microscope. Indeed, a small $h / p$ ratio means that the cholesteric is strongly confined, and that the anchoring overcomes the twist entirely: the cholesteric unwinds, resembling a nematic phase uniformly oriented perpendicularly to the shell interfaces, i.e. radially. Shells with a larger average thickness possess a Janus nature, as shown in Fig. 1b - Fig. 1d. They have a radial director for latitudes such that $h / p<1$ and twisted features for latitudes such that $h / p>1$ : while chirality is repressed around the North pole, it is expressed around the South pole. Indeed, at large local thicknesses, $h / p$ is large, and the cholesteric is weakly confined: the twist is pushed away from the boundaries into the bulk of the shell, and the volume of cholesteric material remains locally twisted.

Shells that are barely too thick to have a radial director everywhere have a small South pole domain where $h / p \gtrsim 1$. Structures of localized twist are confined within this domain: one can observe cholesteric fingers and axisymmetric structures, as shown in Fig. 1b, where the former are indicated with green arrows and the latter with red circles. The axisymmetric structures can consist of various solitonic configurations, such as skyrmions, torons, or hopfions, depending on anchoring or elastic constants. While the delicate nature of shells precluded three-dimensional imaging typically needed to unambiguously identify these structures, ${ }^{49}$ numerical modeling of director field configurations under conditions matching those in experiments revealed their possible nature, as discussed below.

Thicker shells display a striking boundary between twisted and untwisted liquid crystal states, at the latitude where $h=p$. Slightly thicker shells, such as the one shown in Fig. 1c, display a cholesteric cap embedded on an otherwise radial shell, from which extend cholesteric fingers. Besides, one can clearly see the transition between periodic fingers, in the center of the cap, and isolated fingers, in the periphery of the cap, which was described in the previous literature. ${ }^{50,51}$ Much thicker shells also display a twisted/untwisted boundary, but in the northern hemisphere. This leads to a shell that is mostly twisted, except for a featureless radial patch around the North pole, towards which extend stubby fingers, as shown in Fig $1 \mathrm{~d}$ (the location of the fingers is highlighted with blue arrows).

To expand our understanding of the nature of these structures beyond polarizing microscopy imaging, we turn to numerical simulations. We rely on an established numerical technique, based on a randomized director field, generated by way of radial basis functions, and iteratively minimized with a finite-difference method, until a stationary state was reached. ${ }^{46}$ The computational volumes are generated using a technique with nested spheres (see Numerical Methods). They were shaped as asymmetric spherical shells with a finite minimum thickness at the North pole $\left(h_{\min }\right)$ given by the mesh size of the numerical method. We varied the confinement ratio at the South pole of the simulated shells, keeping the pitch $p$ constant, which set the maximum thickness $\left(h_{\max }\right)$. The shells were initialized 

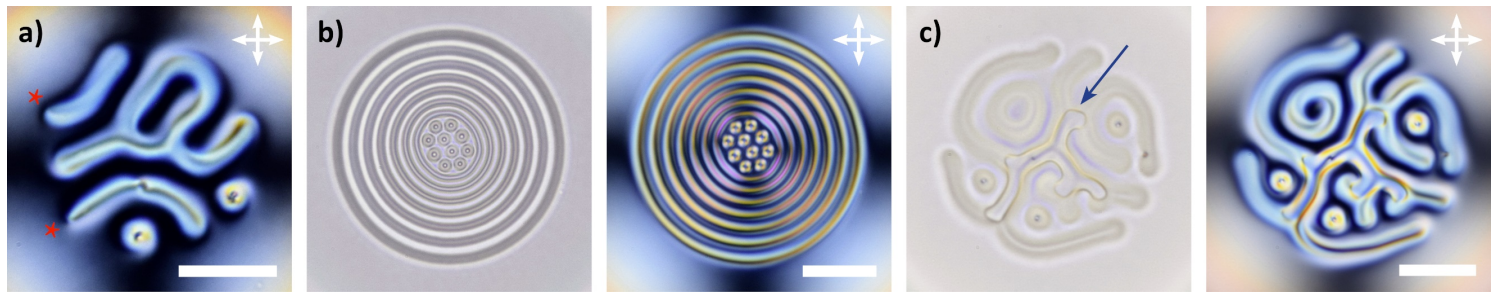

Fig. 3 Diversity of singular and nonsingular structures within cholesteric shells. Bright field (b, left and c, left) and polarized microscopy (a, b, right and c, right) close-ups of the South polar region of shells. a) Non-singular cholesteric fingers and torons on a shell undergoing osmotic swelling. Asterisks denote the pointed end of two CF-1s. b) Looped structures on a shell after a temperature quench from the isotropic phase. c) Looped disclination line, indicated by an arrow, on a shell after an anchoring transition from hybrid to matching perpendicular anchoring. Also featured are one branched cholesteric finger (with ten rounded ends), one looped finger, and three axisymmetric structures. Throughout, scale bars are $25 \mu \mathrm{m}$. White double arrows denote crossed polarizer orientations.

in a random director configuration, seeded with seven singular hedgehog-point-like excitations in the initial director field at the South pole, and left to anneal. This process, which generates seven hexagonally-packed torons under the right confinement ratio, is similar to the annealing procedure used in previous computational works. ${ }^{43,46}$

Fig. 2 presents the results of the simulation of a shell with a local confinement ratio at the South pole $h_{\max } / p=1.25$. A vertical cross-section of the director configuration in the shell is shown in Fig. 2a. Small blue lines with black dots represent the average local orientation of the director field, while black lines with red dots represent the fixed nematic orientation along the edge of the shell, where strong perpendicular surface boundary conditions are enforced. One can distinguish swirls and elongated vortices in the director in most of the southern hemisphere, as well as a uniformly radial northern hemisphere. In order to reveal the director structures effectively, we used additional types of visualizations. In Fig. 2b, we only plot the regions of high twist as purple markers, within the boundaries of the shell represented in yellow, for a side (left panel) and top (right panel) view of the shell. We witness that high twist distortions localize either in linear or ball-like regions, similarly to experiments. Additionally, using a Jones matrix method, ${ }^{6,43}$ we simulate the polarized microscopy textures corresponding to a top view of the shell (Fig. 2c), which qualitatively matches the experiments. We note that the simulated polarizing image matches experimental elementary torons closely but exact comparison to cholesteric fingers is qualitative in nature, which is to be expected due to the inherent limitations associated to the Jones-matrix method. Yet, this method is constantly being improved for highly confined systems with length scales approaching the wavelength of imaging light ${ }^{52}$. Lastly, we sliced through the regions of localized twist, in a series of cross-sections of the southern hemisphere (Fig. 2d): the director field is vectorized and visualized by means of arrows colored according to orientation. This enables us to identify the director configurations in the linear and ball-like twisted regions: consistently with previous works, we recognize cholesteric fingers of the first and second types on the one hand, and skyrmions and elementary torons on the other hand. . $^{73,46}$

Elementary torons are skyrmion tubes terminated on point defects close to confining surfaces. ${ }^{5,17}$ Taking a cross-section through the middle plane of a toron yields a baby skyrmion structure - the twodimensional topological analogue of a nuclear physics skyrmion. ${ }^{1}$ The three-dimensional structure of a toron, spanning through the entire liquid crystal thickness, depends on various experimental parameters, such as the nature of confinement, the strength of surface boundary conditions or the value of the elastic constants. ${ }^{17}$ For these and related structures, the collective, ${ }^{53}$ rotational, 54,55 and translational ${ }^{43}$ motions, optical ${ }^{56,57}$ and transport ${ }^{46}$ properties, self-assembly within chains, ${ }^{58}$ loops, ${ }^{59}$ arrays ${ }^{59-61}$ and clusters, ${ }^{62}$ and interaction with colloidal particles ${ }^{63}$ have been the focus of detailed experimental studies, mostly conducted in solid wedge-shaped and flat glass cells, and rarely in other geometries, namely microchannels $s^{64,65}$ and droplets. ${ }^{66,67}$

Cholesteric fingers of the first ${ }^{14}$ (CF-1s) and second ${ }^{10,18}$ (CF-2s) types are linear regions of twist about one cholesteric pitch wide, which contain line singularities in the pitch, but not in the director. ${ }^{4}$ As both types do not contain line singularities in the director (only in the cholesteric pitch), we will refer to either of them as "non-singular fingers" in the following. They can adopt different morphologies, as shown in Fig. 3a. If their ends meet, they form a loop; if one of their ends splits up, they branch out, leading to a dendritic finger. Fig. $3 a$ features a finger that is both looped and branched. CF-1s can be typically distinguished from CF-2s by their appearance in polarizing micrographs - the former have one pointed end and one rounded end, while the latter have two identical ends - as well as by their dynamics - upon shrinking longitudinally, the former smoothly disappear into the uniform homeotropic texture, while the latter transform into an axisymmetric toron-like structure. ${ }^{3}$ The pointed ends of the two CF1s present in Fig. 3a are indicated by red asterisks. Looped CF-1s cannot shrink indefinitely and disappear: below a given radius, they form an axial particle-like structure. ${ }^{17,68}$ Looped CF-2s disappear when they shrink without forming an axial structure, unless there is a director defect somewhere on the loop. ${ }^{10,68}$

In our experiments, we also observed structures that could not be captured in simulations and might be kinetically stabilized as metastable configurations. Firstly, we observed thin, birefringent linear structures, which, like non-singular cholesteric fingers, are embedded in untwisted domains, but unlike them, are significantly thinner than a pitch. Based on their appearance, we hypothesize that they could be cholesteric fingers of the third type ${ }^{4,6,69-71}$ (CF-3s) or twist domain walls ${ }^{72,73}$. CF-3s and twist walls are related director configurations; their only difference is that two singular twist disclination lines running close to the interfaces are present in CF-3s and not in twist walls. The difference between the two could not be probed here, due to the limited vertical resolution of conventional polarizing microscopy imaging. Small loops of these structures proved to be stable localized particle-like director excitations: Fig. 3b shows both in bright field and polarized microscopy (left and right panels, respectively) a cluster of stable small loops surrounded by larger, nested 
loops. Lastly, sharp lines were found within twisted cholesteric domains, such as the one indicated by an arrow in Fig. 3c. These lines are especially apparent in bright field microscopy and have an optical texture similar to that of nematic disclination lines.

Having first characterized in static situations the global properties of cholesteric shells under strong perpendicular anchoring as well as the nature of the topological solitons and singular defect lines that can spontaneously form inside those shells, we now focus on inducing dynamical interconversions between these delicate structures.

\subsection{Dynamical interconversions between structures of localized twist}

\subsubsection{Transformations between cholesteric fingers and other lin- ear structures}

In traditional solid wedges, the position of the walls is fixed and the magnitude of the anchoring is set by the surface treatment of the walls; both parameters rarely change with time, except in a few elaborate experimental setups ${ }^{74,75}$. All of this leads to spatially distributed textures which cannot evolve with time. Thus, in most setups, the only available means to induce dynamical transitions is to apply external fields to the samples, such as electric, magnetic, or temperature fields. In the case of liquid crystals with a positive dielectric anisotropy, i.e. whose molecules tend to align parallel to the direction of an electric field, this further unwinds the cholesteric helix. In the case of highly confined systems, the anchoring is felt throughout the bulk of the sample, thus inducing a bulk reorientation effect, similarly to external fields. In the case of a shell, however, fluid interfaces enable a much greater tunability of the boundary conditions, making the study of dynamical transitions between textures possible without requiring external fields. We can play with both the alignment and the confinement, as surfactants can adsorb or desorb at the interfaces of a shell, varying the anchoring strength, or as a shell can be swollen or de-swollen, varying its thickness. Besides, while degenerate planar anchoring is possible on a solid wall, this is typically associated to surface friction and memory effects. ${ }^{76}$ With fluid interfaces under planar anchoring, the molecules are free to rotate without these two effects being present. In the following, using this richer experimental playground to our advantage, we report our ability to induce various dynamical interconversions between cholesteric fingers and other linear structures.

Dynamical studies on the nature of the thin linear structures. In a first series of dynamical experiments, documented in Fig. 4, we showed that the thin linear structures reported at the end of the previous section are related to $\mathrm{CF}-3 \mathrm{~s}$.

We first showed that a disclination could guide the formation of such thin linear structures and be eventually replaced by it - thus supporting the CF-3 hypothesis, as these objects are the simplest fingers which include line defects of the director. To do so, we performed a slow anchoring transition on cholesteric shells, shown in Fig. 4a. Here, the shells were initially produced without SDS, neither in their inner water droplet nor in the continuous water phase. They were then re-dispersed in a water solution with $10 \mathrm{mM} \mathrm{SDS}$. The anchoring quickly changed from planar to homeotropic on the outer interface, leading to hybrid anchoring conditions in the early stages of the experiment. As the surfactants slowly diffused through the liquid crys-

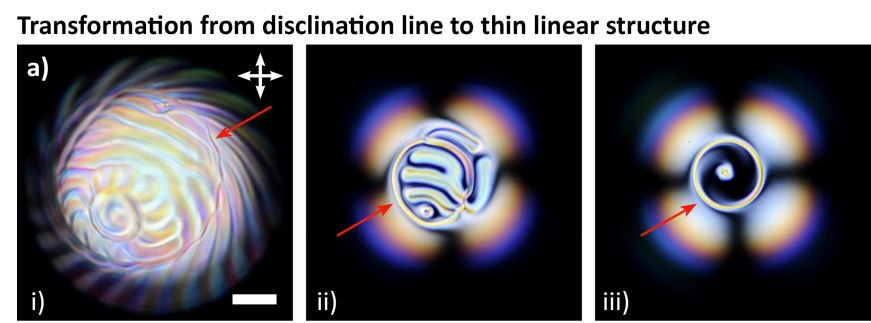

Decay of thin linear structures upon cooling
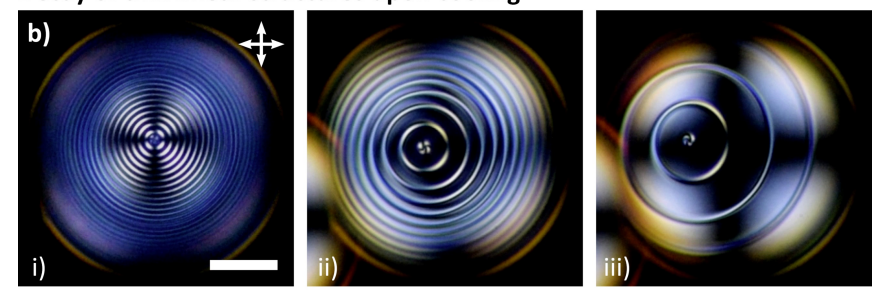

Static interactions between nonsingular fingers and a thin linear structure
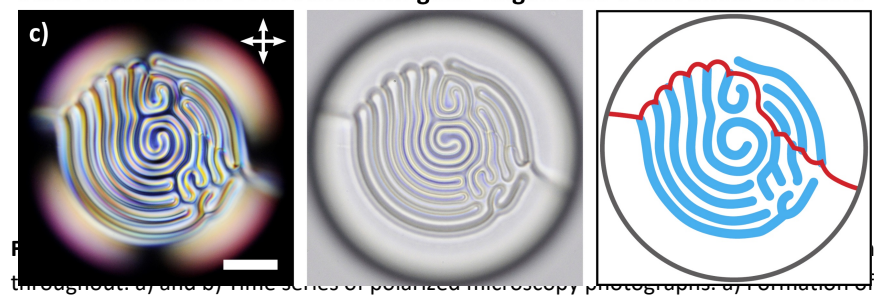

a looped structure during an anchoring transition from homeotropic inside and planar outside, to homeotropic both inside and outside. i) A sharp disclination loop, identified with an arrow, is visible in the cholesteric texture. ii) During the shell evolution, the disclination loop seems to be replaced by a looped birefringent structure, also identified with an arrow, from which non-singular fingers grow. iii) At the end of the transition, only this loop and an axisymmetric structure remain. b) Decay of a large number of looped structures created from a temperature quench. i) About a hundred milliseconds after the nucleation of the cholesteric phase, the South pole of the shell gets covered with concentric loops that are spaced half a pitch from one another. ii) These loops quickly decay one after the other by shrinking down to a point. iii) After a few tens of seconds, only a few loops remain. c) Non-singular fingers anchoring a disclination line to the thick part of a shell. Polarized microscopy (left), bright field microscopy (middle) and sketch of the structures (right). The pictures show the South pole of a shell with a very thin cholesteric cap. A disclination line, sketched in red, wraps around the shell. Cholesteric fingers, sketched in blue, anchor it to the South pole. White double arrows denote crossed polarizer orientations.

tal membrane, the anchoring was also affected on the inner interface, going from planar to homeotropic in about a day. Therefore, such a slow-paced anchoring transition enabled us to follow the temporal evolution of the structures on the shells. Initially, shells displayed a thick-stripe texture, ${ }^{30}$ as shown in Fig. $4 a-i$, indicating the bending of the cholesteric layers to accommodate both homeotropic and planar cues at the outer and inner interfaces, respectively, in a relatively thick shell. The texture also featured one or several disclination loops, such as the one indicated by an arrow. Then, as SDS molecules kept adsorbing onto the inner interface, and as the cholesteric was slowly reorganizing to adapt to the full homeotropic anchoring cues, the twisted cholesteric was abruptly replaced by unwound cholesteric, and the disclination loop by a looped thin linear structure (indicated by an arrow in Fig. 4a-ii), from which stemmed non-singular cholesteric fingers. At the end of the transition, the initial disclination seemed to have been entirely replaced by the new structure (Fig. 4a-iii). This indicates that both are likely to be two 
manifestations of similar underlying director configurations, depending on whether they are embedded in twisted or unwound domains, respectively.

We then established the energetically-costly nature of these thin linear structures by performing temperature quenches on Janus shells with a small cholesteric cap. Upon cooling them down abruptly from the isotropic to the cholesteric phase, we witnessed the formation of a great number of such looped structures, as recorded in Supplementary Movie 1 (real-time film). Fig. $4 \mathrm{~b}$ shows the evolution of such a shell a hundred milliseconds after the nucleation of the cholesteric phase. The initial texture was a very regular arrangement of loops, located half a pitch from one another, and organized in concentric circles (Fig. 4b-i). Additional observations of the onset of the phase transition in bright field microscopy showed that the cholesteric phase nucleates first at little impurities within the shell, such as small satellite water droplets encapsulated within the liquid crystal membrane, and that these nucleation points govern the initial arrangement of nested loops. An easier formation of these structures at higher temperatures is consistent with their ability to induce costlier singular distortions. For temperatures in the vicinity of the clearing point, elastic constants are weaker, and strong distortions are less energetically costly. As the system cooled down rapidly, the loops were observed to shrink, reducing their length, until they disappeared into a point one after the other (Fig. $4 \mathrm{~b}$-ii). In less than a minute, all but a couple of loops had disappeared (Fig. 4b-iii).

Interestingly, while singular structures are usually transient features of a shell, we showed that they could endure when non-singular fingers are attached to them. This is evidenced in Fig. 4c. It depicts a shell encircled from North to South pole by a disclination structure (sketched in red). The cholesteric cap around the South pole is made of disjoined non-singular cholesteric fingers (sketched in blue). The fingers grow from kinks in the disclination, on either side of it, and pack as a roughly circular cap. This leads to an unusual behavior for a disclination-like structure on a shell, since they are usually located at the thinnest region of the shell in order to reduce the volume of material affected by their presence. As the fingers are located at the thicker South pole, they anchor the line defect in a thick region where it would otherwise be too costly.

\section{Dynamical interconversions between non-singular cholesteric fin-} gers and thin linear structures. In a second series of dynamical experiments, detailed in Fig. 5, we focused on how non-singular cholesteric fingers can be transformed into those thin linear structures, and vice versa.

In a first experiment, we dynamically increased the confinement in a Janus shell of a cholesteric liquid crystal with a small pitch, through a monotonous decrease of its thickness with time. We produced thin shells with a $2.35 \mu \mathrm{m}$ pitch, half the value used throughout the rest of this study. We enforced strong homeotropic boundary conditions as usual, with a $10 \mathrm{mM}$ SDS concentration both inside and outside the shells, which displayed a cholesteric cap bounded by small fingers. Additionally, a $1 \mathrm{M} \mathrm{NaCl}$ concentration difference was imposed between their inner water droplet and the outer continuous water phase, which set an osmotic pressure difference between the two water phases. Indeed, liquid crystal shells act as spherical membranes separating the inner from the outer phase, permeable to water but impermeable to salts. Therefore, a high $\mathrm{NaCl}$ concentration in the inner droplet drives a flux of water from the outer solution

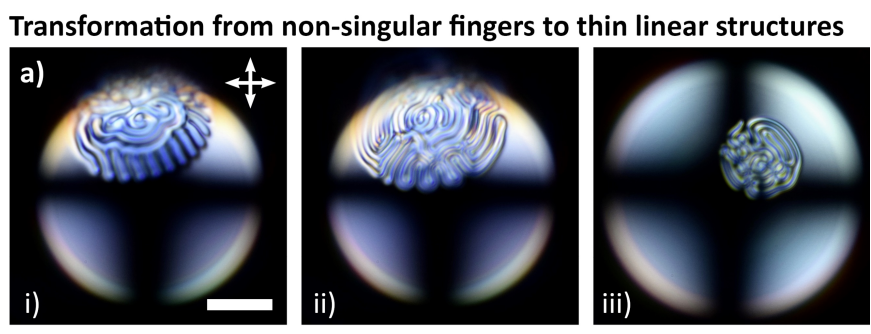

Transformations from thin linear structures to non-singular fingers
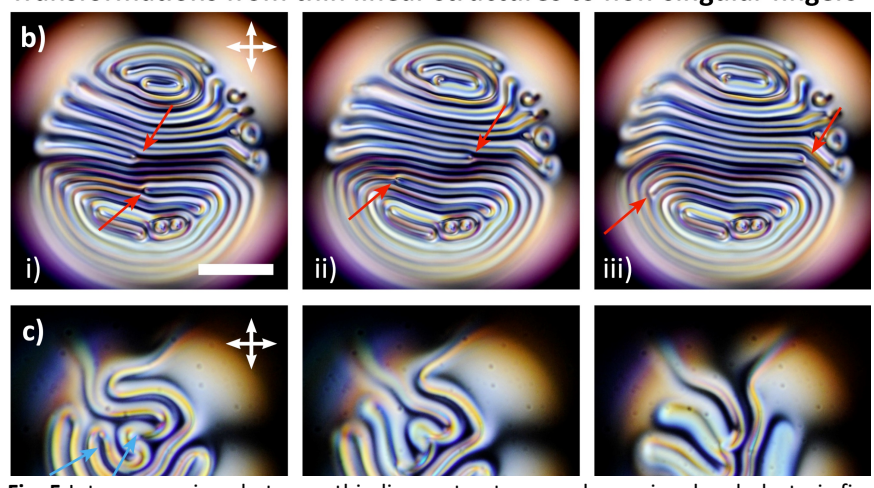

Fig. 5 Interconversions between thin linear structures and non-singular cholesteric fingers. Time series of polarized microscopy photographs. Throughout, scale bars are 25 $\mu \mathrm{m}$ and white double arrows denote crossed polarizer orientations. a) A shell with a small cholesteric pitch is osmotically swollen. i) Initially, the shell has a cholesteric cap with non-singular fingers. ii) It transforms abruptly into a bundle of individual thin linear structures iii) that eventually shrink and disappear. b) Thin linear structures "unzipping" into non-singular fingers on a shell relaxing after a temperature quench. At the location indicated by the arrows, the thin structures are being progressively replaced by non-singular fingers. c) Curls in thin linear structures being transformed into non-singular cholesteric fingers, at the location indicated by the arrows, on a shell undergoing an anchoring transition from hybrid to matching planar anchoring.

through the liquid crystal, osmotically swelling the shell until the concentrations on either sides equilibrate. ${ }^{25,28,77}$ As the salty water is denser than the liquid crystal, shells in osmotic swelling experiments are "flipped" upside down, i.e. their North and South poles are respectively the points of largest and smallest local thicknesses. Swelling experiments last about one hour and are characterized by an abrupt transformation of the entire cap from cholesteric fingers to a bundle of thin linear structures, as recorded in Supplementary Movie 2 (time-lapse, sped up 225 times), from which Fig. 5a-i and Fig. 5a-ii are extracted. Then for even higher confinement values, the new structures gradually shrink until none are left and the shell becomes entirely radial.

In another series of experiments on shells with the usual pitch of 4.7 $\mu \mathrm{m}$, we observed the reverse transformation, that took place spontaneously in different modalities. Thin linear structures produced in large numbers following a temperature quench were observed a few times to "unzip" from one end into a regular cholesteric finger. This phenomenon is occurring simultaneously on two separate instances, indicated with an arrow, in the sequence of polarized microphotographs that constitutes Fig. 5b, extracted from real-time Supplementary Movie 3. Another mode leading to the destabilization of thin linear structures into a non-singular cholesteric finger is illustrated in Fig. $5 \mathrm{c}$ : these structures were often observed to bend and buckle into series of narrow curls; at some point, the two sides of the curl would 

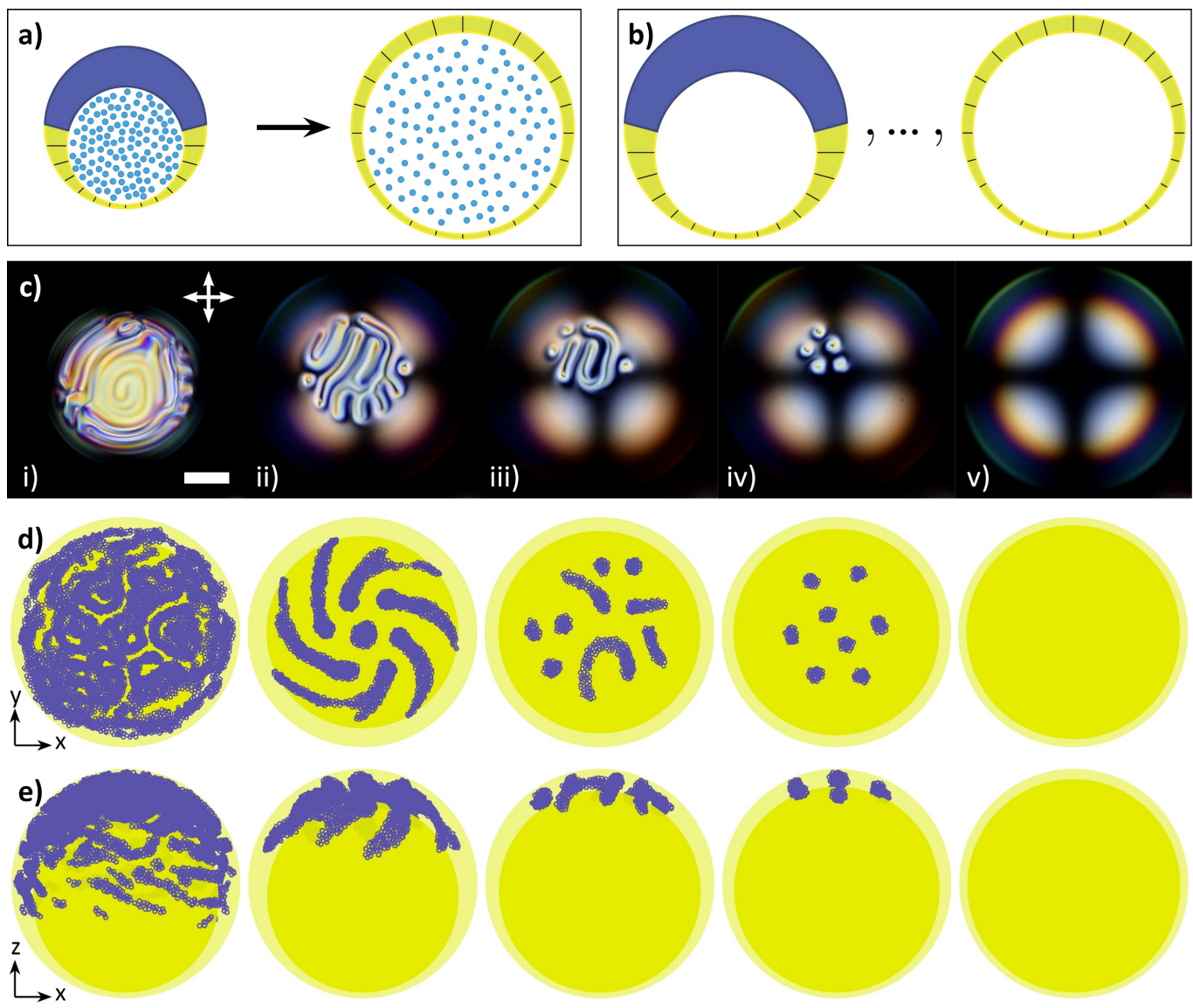

Fig. 6 Sweeping through shell textures using osmosis. a) Side-view schematics indicating the principle of the experiment (a) and of the simulations (b). Yellow: boundaries of the shell, purple: twisted cholesteric domain, blue circles: salt. In a), a shell swells over time due to a higher salt concentration in the inner water droplet than in the continuous water phase, until the two equilibrate. In b), shells with a fixed outer radius and an increasing inner radius are simulated. c) Series of polarized top-view microphotographs of the experiment. Scale bar is $25 \mu \mathrm{m}$ and white double arrows denote crossed polarizer orientations. d) (resp. e) Series of top-view (resp. side-view) visualizations of the numerical simulations, showing the boundaries of the shell (yellow) and the regions of high twist distortions (purple markers), for $h_{\max } / p=2,1.4,1.1,1.0$ and 0.7 .

merge with one another, becoming a non-singular finger in the process. The specific event recorded in Supplementary Movie 4 (timelapse, sped up 75 times), from which was extracted Fig. 5c, occurred during an anchoring transition experiment, without swelling or temperature changes.

\subsubsection{Targeted production of axisymmetric structures}

In this last section, we aim to answer the following question: what is the best way to produce an array of axisymmetric structures on a shell? Indeed, among the various dynamical interconversions that we can study within our shells, this is the one that can produce elementary torons or skyrmions, some of the most sought-after topological structures.

Osmotic swelling. We started with an osmotic swelling experiment, whose principle is shown in Fig. 6a and whose results are recorded in Supplementary Movie 5 (time-lapse, sped up 75 times) and shown in Fig. $6 \mathrm{c}$. Janus shells of the regular $4.7 \mu \mathrm{m}$ pitch cholesteric mixture were produced; they displayed a thick cholesteric cap embedded on a mostly radial shell (Fig. $6 \mathrm{c}-\mathrm{i}$ ). The $\mathrm{NaCl}$ concentration was $1 \mathrm{M}$ higher in their inner water droplet than in the continuous water phase. Swelling takes place within a time period of about one hour. As the cap becomes thinner, it evolves into a collection of branched CF-2s (Fig. 6c-ii). Then, as the confinement increases, long extended fingers are not stable anymore and shrink longitudinally (Fig. 6c-iii), yielding one toron/skyrmion each, which pack at the thick pole of the shell as a little cluster (Fig. 6c-iv). Eventually, when the local thickness becomes too small, they disappear abruptly, leaving behind a featureless radial shell (Fig. 6c-v).

This experiment thus has a strikingly different result from the one with a $2.35 \mu \mathrm{m}$ pitch (Fig. 5a). Indeed, to form a cap with a smaller pitch, one needs a shell that is on average thinner, and thus, more uniform in thickness. We hypothesize that the difference in the outcome of the two experiments comes from the difference in the nature of the confinement.

To gain a better understanding of our experimental observations, we simulated the various stages of this evolution. The simulated shells had a fixed outer radius and increasing values of the inner radius reproducing the growing levels of confinement in the experiments (Fig. 6b). Torons and various twisted structures were generated on the computational shell with the procedure described above. This means that a shell with $h_{\max } / p=1$ forms stable torons, as they correspond to a minimum in the distortion energy, while in thicker or thinner shells, these point-like excitations are unstable, and 

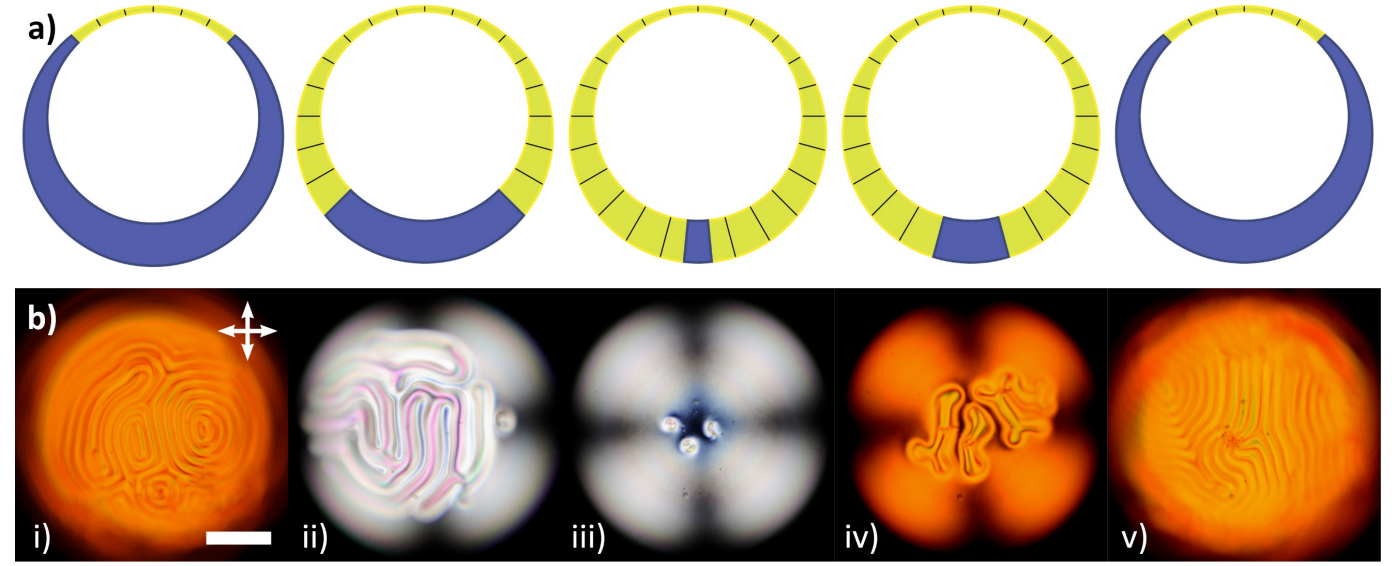
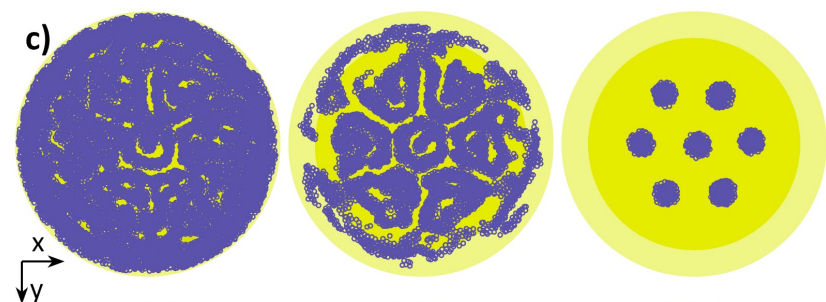

d)
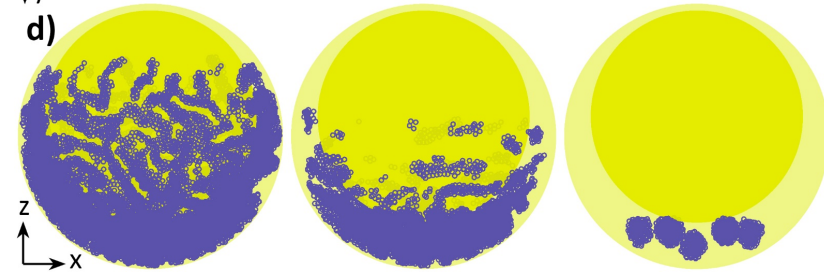
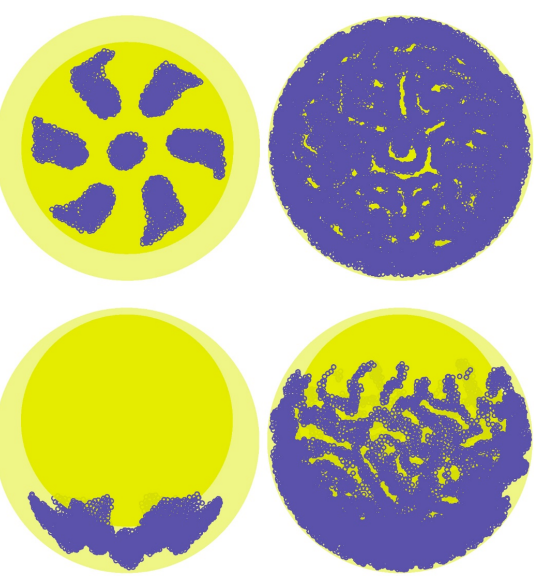

Fig. 7 Transitioning from twisted cholesteric to torons using pitch phototuning. a) Series of side-view schematics showing the principle of the experiment. Yellow: boundaries of the shell, purple: twisted cholesteric domain. b) Series of polarized top view microphotographs. i), iv) and v) are under orange illumination, which induces a small value of the cholesteric pitch; ii) and iii) are under white illumination, which yields a larger pitch. i), iii) and v) are equilibrium states; ii) and iv) are transitory states. Scale bar is $25 \mu \mathrm{m}$ and white double arrows denote crossed polarizer orientations. c) (resp. d) Series of top-view (resp. side-view) numerical simulations displaying the regions of localized twist (purple markers) within the shell boundaries (yellow), for $\frac{h_{\max }}{p}=3.5,3,1,1.4$ and 3 .

morph into other director structures. For local confinement ratios equal to $h_{\max } / p=2,1.4,1.1,1.0$ and 0.7 , simulated shells display a director configuration represented in Fig. $6 \mathrm{~d}$ (side view) and Fig. $6 \mathrm{e}$ (top view). These isolated snapshots do capture the different stages of the dynamical transition.

In practice, a swollen shell cannot be de-swollen: it would require replacing the surrounding continuous phase by a new solution with a different salt concentration, without losing track of the shell. Thus osmotic swelling can be considered as an irreversible process. It is also very difficult to target a final confinement ratio with a precise value with this technique. Therefore, it is appropriate when one wants to sweep through all the possible stages of a dynamical transition, but not when one is willing to stabilize one stage in particular. Pitch phototuning. We turned to dynamically modifying the value of the pitch $p$, which constitutes a different way to tune the confining ratio $c=h / p$. We used a phototunable molecule known as OA-1; it is an overcrowded alkene, a class of molecules that has already been employed as molecular motors or phototunable chiral dopants. ${ }^{39-42}$ Under UV or blue light illumination, the molecule slowly isomerizes to an excited conformation with a different helical twisting power. In about fifteen minutes, a photo-stationary state is reached, and the equilibrium pitch of the cholesteric is increased with respect to the initial state. When the blue light is turned off, the molecules quickly relax to their ground state, a process that typically takes about one minute. Therefore, we expected to be able to transition between two stable textures: one at low confinement, induced by a small pitch when the blue light is off, and a second one at high confinement, induced by a large pitch when the blue light is on - similarly to other studies using pitch phototuning to manipulate various structures of localized twist ${ }^{55,61,78-81}$.

Experimentally, shells of the phototunable mixture were produced in water solutions of SDS, in such a way that their local thickness at the South pole matched the excited value of the pitch. The photo-induced evolution of one of these shells is shown in Fig. 7b. To observe the ground state, the blue wavelengths of visible light are blocked by an orange filter; removing the filter induces a transition to the excited state, as shown in Supplementary Movie 6 (time-lapse, sped up 345 times). Initially, the shell displays a twisted cholesteric texture, consistent with a weak confinement (Fig. 7b-i). Removing the filter induces a slow transition to a larger value of the pitch. Then a transitory texture with shrinking branched cholesteric fingers is observed (Fig. 7b-ii), before a photo-stationary state is reached after a couple hours. As the maximum thickness of the shells matches the new value of the pitch, the shell features elementary torons, which are stable structures for $h / p \approx 1$ (Fig. 7b-iii). They last as long as the white light illumination is maintained, as shown in Supplementary 
Movie 7 (real-time). Introducing the orange filter again induces a quick growth of dendritic fingers from the torons (Fig. 7b-iv), until the twisted cholesteric texture is recovered (Fig.7b-v), in about two minutes.

To gain a better insight of the transition between these structures, we also computer-simulated its various stages. Using the same parameters as before, we simulated a series of shells with a fixed geometry (both $h_{\min }$ and $h_{\max }$ were constant across the series). We varied the local confinement ratio at the South pole, thus setting the value of the cholesteric pitch for each shell - mimicking the experimental photo-induced change in pitch values. The shells were initialized in a random director configuration, seeded with seven point-like excitations in the initial director field, and left to anneal. Our results are shown in Fig. 7c and Fig. 7d, corresponding to confinement ratios of $h_{\max } / p=3.5,3,1,1.4$, and 3.5. A confinement ratio of 3 , corresponding to a small value of the pitch, yields regions of high twist spread out across the thick part of the shell, similarly to the twisted cholesteric cap found in experiments. For a confinement ratio equal to 1 , torons appear from the initial excitation regions and adopt wellrounded shapes. We also capture structures corresponding to transitory states in our experiment (confinement ratios of 1.4 and 3 ), which are "spread-out" fingers and "extended" torons.

Although reversible, the manipulation of shell textures through pitch phototuning can only enable us to stabilize two discrete states in a given experiment, and switching between them typically takes either a minute or an hour. We therefore turned to electric fields to bring an even finer control over the textures.

Electric field unwinding. Electric fields unwind the cholesteric helix, promoting molecular alignment along the field for liquid crystals such as $5 \mathrm{CB}$, with a positive dielectric anisotropy. Applying a vertical electric field on a shell promotes molecular reorientation in the vertical direction. Therefore, at the poles of the shell, it should favor a radial, untwisted director field, while at its equator, it should oppose the presence of a radial field, hence promoting twisted structures. But the shell thickness decreases with the altitude, and with it, the amplitude of the electric field required to overcome the elasticity of the liquid crystal increases too. Thus, by keeping the field amplitude low enough, we only reorient the liquid crystal molecules in the thickest part of the shell, close to the South pole, and not in the rest of the shell. Experimentally, thin shells of the regular 5 CB/CB15 mixture with a $4.7 \mu \mathrm{m}$ pitch were produced, in such a way that their South pole displayed disjoined fingers, the rest of the shell being radial. The shells were submitted to a vertical high-frequency electric field for short periods of time by means of an observation chamber equipped with transparent ITO electrodes (Fig. 8a); the typical response of a shell is given in Fig. $8 \mathrm{~b}$ and in real-time Supplementary Movie 8.

Before the field is applied for the first time, shells display a thin cholesteric cap at their South pole, filled with disjoined cholesteric fingers, such as the one shown in Fig. 8b-i, made of a single branched finger. As soon as the field is turned on, the fingers retract, transforming to one axisymmetric structure per branched finger in a few seconds (Fig. 8b-ii). Those structures are stable as long as the field is on: upon turning it off, the fingers grow back immediately, reaching a constant size only after having filled the volume of the initial cholesteric cap (Fig. 8b-iii). Although the area covered by the fingers remains the same, the details of their shapes are now different, indicating that the system can be switched between various metastable states. The transition from fingers to axisymmetric structures can be repeated at will (Fig. $8 \mathrm{~b}$-iv and Fig. $8 \mathrm{~b}-\mathrm{v}$ ). In addition to being nearly instantaneous, this field-induced transition is also continuous: one can stabilize the twisted structures at any stage between the extended fingers and the axisymmetric structures, simply by imposing a field with a smaller amplitude.

Lastly, although all samples were produced the same way, some of them had a different response to the field: in some samples, shells were observed to translate along random in-plane directions, while in other samples, shells were seen to rotate by $90^{\circ}$ around an inplane axis, positioning their cholesteric cap where the equator of the shell used to be. We interpret the former situation as a result of flows induced in the continuous water phase, and the latter as a consequence of the tendency of the field to promote non-radial director configurations at the equator of a shell.

Excursions through the isotropic phase. All of the experimental techniques employed so far proved useful for the investigation of dynamical transitions between twisted structures, but were unable to produce large numbers of axisymmetric structures. How can we produce them in large numbers on a shell that has the adequate thickness to accommodate them? Conversely, how can we erase them when they are already embedded in a shell? As metastable structures, they cannot be created or destroyed in a director field that is everywhere well-defined at all times unless singular defects mediate such trans-

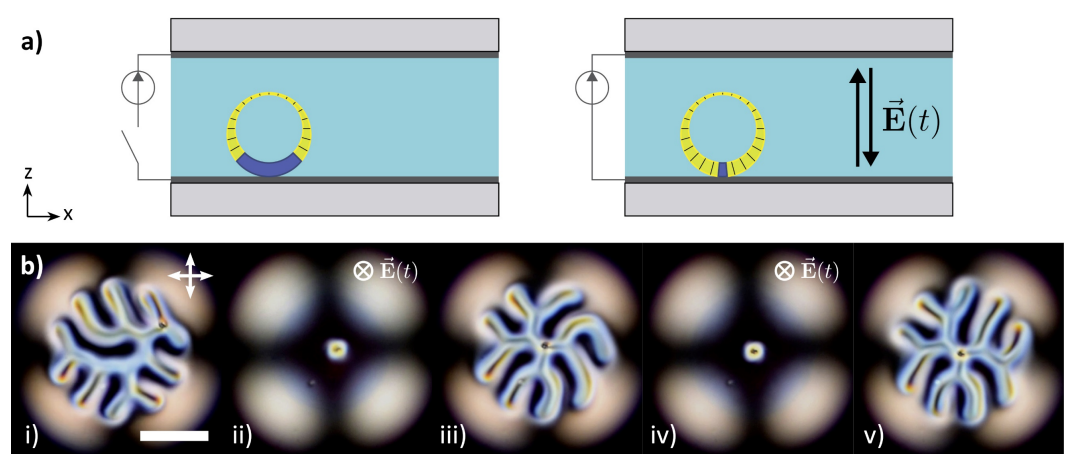

Fig. 8 Transitioning from cholesteric fingers to axisymmetric structures using an electric-field-induced helix unwinding. a) Experimental setup. A $200 \mu \mathrm{m}$ thick observation chamber is made of two ITO-coated glass slides, enabling the vertical application of alternating electric fields on thin cholesteric shells. The field promotes the retraction of the twisted cholesteric texture at the South pole (purple) in favor of a director oriented parallel to the field. b) Series of polarized top view microphotographs showing the texture when the field is turned off ( $\mathrm{i}, \mathrm{iii}, \mathrm{v}$ : branched cholesteric finger) or turned on (ii, iv: axisymmetric structure). Scale bar is $25 \mu \mathrm{m}$, white double arrows denote crossed polarizer orientations, and the direction of applied alternating electric field $\overrightarrow{\boldsymbol{E}}(\boldsymbol{t})$ is marked in white on relevant frames. 
formations. However, a phase transition, creating pockets of isotropic phase where the director is not defined, can be used for this purpose. In the following, we consider the effects of three different experimental techniques to induce a phase transition in the shell.

For this experiment, cholesteric shells were produced at the thickness for which a few axisymmetric structures spontaneously appear at their South pole. The effect of nucleating localized pockets of isotropic phase on the shells was first investigated, as suggested by the literature. ${ }^{17}$ The temperature of the system was slowly raised in the vicinity of the clearing point of $5 \mathrm{CB}$ using a precision oven, until the nucleation of the first isotropic spots on the shell. At this point, the system was cooled down immediately. Axisymmetric structures were created while the pockets of isotropic phase collapsed on themselves, but the delicate stability of the thin liquid crystal membrane could not withstand the transition back to an ordered phase, and shells were seen to burst every time.

When similar shells were placed into observation chambers that were equipped with ITO electrodes and subsequently subjected to an alternating electric field, we witnessed three types of responses from the shells, depending on the amplitude and the duration of application of the field. When fields are applied for a short time with a low amplitude, the axisymmetric structures are observed to shrink; they grow back to their original size when the field is turned off. Essentially, this is the same reversible unwinding of the cholesteric helix as previously reported in the case of the fingers (Fig. 8). When fields are applied for a short time above a certain amplitude, however, an energy barrier is overcome and the cholesteric helix unwinds itself entirely: the field is strong enough to erase the structures irreversibly. Conversely, applying a low-amplitude field for several tens of seconds turned out to be a reliable mechanism to create axisymmetric structures on the shell. Indeed, this drives the system up to the isotropic phase, as reported in a recent study. ${ }^{82}$ Axisymmetric structures can then be generated by turning off the field immediately after the nucleation of the first isotropic patches. Contrary to the oven, the shells are not affected and do not burst, likely due to the smaller response time of this unusual heating technique. This mechanism, shown in Fig. 9 and recorded in real-time Supplementary Movie 9, was therefore very useful in creating arbitrary numbers of axisymmetric structures on a shell. It is important to remember that throughout all our experiments, shells sediment to the bottom of the observation chamber: in this case, they rest directly on the ITO layer. Lastly, shells of a 2:1 mixture of cholesteric liquid crystal and chloroform were produced with a similarly small thickness. As chloroform disrupts the liquid crystalline order, these shells were formed in an isotropic liquid state. Yet as chloroform leaves the shell, dissolving in the water around it, shells revert to the cholesteric state, nucleating in little localized pockets throughout the shell, at the thickness for which axisymmetric structures are stable. Thus, with such a slowpaced, gentle, and disordered phase transition, we create hundreds of them at once. Beyond the technical achievement they represent, these methods produce an experimental system in which particlelike structures pack within a curved space, therefore constituting a promising candidate for conducting spherical crystallography studies.
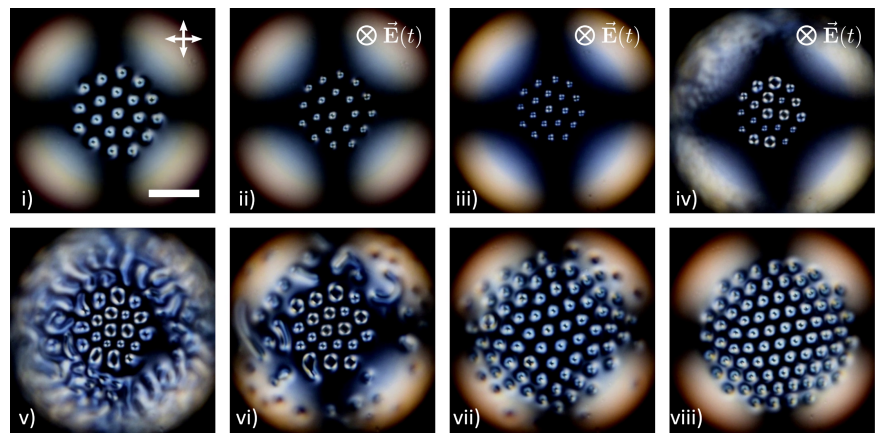

Fig. 9 Creating a multitude of axisymmetric structures on a shell. Time series of polarized microscopy photographs of a shell subjected to an alternating vertical electric field triggering a phase transition. i) Initially, a thin cholesteric shell under perpendicular anchoring has 20 axisymmetric structures packed at its South pole. ii) Upon turning on the field, the radii of the structures shrink. iii) The field is maintained for a dozen seconds as the sample heats up (note the subtle change in the birefringence colors of the shell, indicating that temperatures are approaching the clearing point). iv) As the liquid crystal undergoes the phase transition to isotropic, the radius of the existing structures expand again. v) The field is turned off and the numerous pockets of isotropic phase that just appeared immediately collapse on themselves. vi) As the temperature decreases quickly, the various new cholesteric structures take a more ordered appearance. vii) The newly generated axisymmetric structures gather at the thickest part of the shell, driven to the pole by the thickness heterogeneity. viii) After several rearrangements, all 78 structures arrange in a lattice. Scale bar is $25 \mu \mathrm{m}$, white double arrows denote crossed polarizer orientations, and the direction of applied electric field is marked in white on relevant frames.

\section{Conclusion}

This work constitutes the first study of topological solitons on cholesteric shells under high geometrical confinement and strong perpendicular surface anchoring. We show that the cholesteric texture on such shells splits into a twisted region and a radial domain, giving the shells a Janus nature. At the boundary between the twisted and untwisted domains, various structures of localized twist emerge. In particular, we identify among them non-singular cholesteric fingers, elementary skyrmions and torons, whose complex structures we verify by means of computer simulations using novel numerical techniques. We also report different types of linear structures, showcasing a zoo of topological structures realizable on shells. We take advantage of the versatility of our system to induce a large variety of dynamical interconversions between these structures using a range of experimental techniques. We show the effect of different natures of confinement on the observed structures. We introduce pitch phototuning in shells as an alternative way to tune confinement between two states, with a greater precision than traditional osmosis techniques. We demonstrate that electric fields can also be used to induce interconversions between a continuum of states. In the end, we tackle the targeted production of axisymmetric structures with a finer and finer level of control, and capture the various stages of these transitions numerically. Eventually, we uncover a reliable method to create them in large numbers on a shell, opening the door for the creation of a new model system to tackle spherical crystallography. While our work has demonstrated how various localized cholesteric structures can be controllably created and inter-transformed on chiral nematic shells by means like temperature changes, shell thickness variations, phototuning of pitch and so on, one parameter 
that is key for selecting skyrmions versus torons and walls versus fingers is the strength of surface anchoring. ${ }^{45}$ While anchoring certainly varied with changing all these physical parameters in our work, we did not characterize it due to complexity of the spherical shell geometry, which is outside of the scope of our present study. This, however, poses new opportunities for future studies, where the strength of perpendicular boundary conditions can be systematically tuned and characterized along with parameters like shell thickness, so that the host of solitonic localized structures can be controllably selected to appear on demand and then imaged in 3D, as recently demonstrated for flat cells. ${ }^{45}$

\section{Conflicts of interest}

There are no conflicts to declare.

\section{Acknowledgements}

We thank Timothy Bunning and Timothy White for providing the OA1 phototunable dopant. We thank Lisa Tran, Maxim O. Lavrentovich, Randall D. Kamien, Jung-Shen Tai and Timothy White for the insightful discussions that motivated this study. This work was supported by the Agence Nationale de la Recherche (ANR) through grant No. 13JS08-0006-01, and by the National Science Foundation through grants DMR-1810513 (research), DGE-1144083 (Graduate Research Fellowship to H.R.O.S.) and ACl-1532235 and ACl-1532236 (RMACC Summit supercomputer used for numerical modeling).

\section{References}

N. Manton and P. Sutcliffe, Topological Solitons, Cambridge University Press, Cambridge, 2004. P.-G. de Gennes and J. Prost, The physics of liquid crystals, Clarendon Press, 1993.

3 P. Oswald and P. Pieranski, Nematic and cholesteric liquid crystals: concepts and physical properties illustrated by experiments, CRC Press, 2005.

$4 \quad$ I. I. Smalyukh, B. I. Senyuk, P. Palffy-Muhoray, O. D. Lavrentovich, H. Huang, E. C. Gartland, V. H. Bodnar, T. Kosa and B. Taheri, Phys. Rev. E, 2005, 72, 061707.

5 I. I. Smalyukh, Y. Lansac, N. A. Clark and R. P. Trivedi, Nat. Mater., 2010, 9, 139-145.

6 P. J. Ackerman, R. P. Trivedi, B. I. Senyuk, J. Van De Lagemaat and I. I. Smalyukh, Phys. Rev. E, 2014, 90, 012505.

7 P. J. Ackerman and I. I. Smalyukh, Phys. Rev. X, 2017, 7, 011006.

8 M. Brehm, H. Finkelmann and H. Stegemeyer, Berichte der Bunsen-gesellschaft für Phys. Chemie, 1974, 78, 883-886.

9 T. B. Harvey, Mol. Cryst. Liq. Cryst., 1976, 34, 225-229.

10 J. Baudry, S. Pirkl and P. Oswald, Phys. Rev. E, 1999, 59, 5562-71.

11 S. Pirkl, Cryst. Res. Technol., 1991, 26, K111-K114.

12 W. E. L. Haas and J. E. Adams, Appl. Phys. Lett., 1974, 25, 535-537.

14

15

16

17

18

19

20

23

24

1974, 13, 1457-1458.

M. J. Press and A. S. Arrott, Mol. Cryst. Liq. Cryst., 1976, 37 387-395.

J. Wahl, J. Phys. Colloq., 1979, 40, C3-98-C3-103.

K. Nakamura and N. Nawa, Jpn. J. Appl. Phys., 1978, 17, 219-225.

S. Pirkl, P. Ribière and P. Oswald, Liq. Cryst., 1993, 13, 413425.

L. Gil and J. M. Gilli, Phys. Rev. Lett., 1998, 80, 5742-5745.

A. Fert, V. Cros and J. Sampaio, Nat. Nanotechnol., 2013, 8, 152-156.

N. Romming, C. Hanneken, M. Menzel, J. E. Bickel, B. Wolter, K. von Bergmann, A. Kubetzka and R. Wiesendanger, Science, 2013, 341, 636-639.

N. Nagaosa and Y. Tokura, Nat. Nanotechnol., 2013, 8, 899-911.

A. Fert, N. Reyren and V. Cros, Nat. Rev. Mater., 2017, 2, 17031.

T. Lopez-Leon and A. Fernandez-Nieves, Colloid Polym. Sci., 2011, 289, 345-359.

M. Urbanski, C. G. Reyes, J. Noh, A. Sharma, Y. Geng, V. Subba Rao Jampani and J. P. F. Lagerwall, J. Phys. Condens. Matter, 2017, 29, 133003.

T. Lopez-Leon, V. Koning, K. B. S. Devaiah, V. Vitelli and A. Fernandez-Nieves, Nat. Phys., 2011, 7, 391-394.

H. Poincaré, J. Math. Pures Appl., 1885, 1, 167-244.

H. Hopf, Math. Ann., 1927, 96, 225-249.

A. Darmon, M. Benzaquen, D. Seč, S. Čopar, O. Dauchot and T. Lopez-Leon, Proc. Natl. Acad. Sci., 2016, 113, 94699474.

A. Darmon, M. Benzaquen, S. Čopar, O. Dauchot and T. Lopez-Leon, Soft Matter, 2016, 12, 9259-9392.

L. Tran, M. O. Lavrentovich, G. Durey, A. Darmon, M. F. Haase, N. Li, D. Lee, K. J. Stebe, R. D. Kamien and T. LopezLeon, Phys. Rev. X, 2017, 7, 041029.

J.-Y. Kwon, M. Khan and S.-Y. Park, RSC Adv., 2016, 6, 55976-55983.

J.-H. Jang and S.-Y. Park, Sensor. Actuat. B-Chem., 2017, 241, 636-643.

Y. Uchida, Y. Takanishi and J. Yamamoto, Adv. Mater., 2013, 25, 3234-3237.

Y. Geng, J. Noh, I. Drevensek-Olenik, R. Rupp, G. Lenzini and J. P. F. Lagerwall, Sci. Rep., 2016, 6, 26840.

J.-H. Kang, S.-H. Kim, A. Fernandez-Nieves and E. Reichmanis, J. Am. Chem. Soc., 2017, 139, 5708-5711. S.-W. Ko, S.-H. Huang, A. Y.-G. Fuh and T.-H. Lin, Opt. Express, 2009, 17, 15926-15931.

A. S. Utada, E. Lorenceau, D. R. Link, P. D. Kaplan, H. A. Stone and D. A. Weitz, Science, 2005, 308, 537-541. J. M. Brake and N. L. Abbott, Langmuir, 2002, 18, 61016109.

R. Eelkema, M. M. Pollard, N. Katsonis, J. Vicario, D. J. Broer and B. L. Feringa, J. Am. Chem. Soc., 2006, 128, 14397-14407.

R. Eelkema, M. M. Pollard, J. Vicario, N. Katsonis, B. S. Ramon, C. W. M. Bastiaansen, D. J. Broer and B. L. Feringa, Nature, 2006, 440, 163-163. 
41 A. Bosco, M. G. M. Jongejan, R. Eelkema, N. Katsonis, E. Lacaze, A. Ferrarini and B. L. Feringa, J. Am. Chem. Soc., 2008, 130, 14615-14624.

T. J. White, S. A. Cazzell, A. S. Freer, D.-K. Yang, L. Sukhomlinova, L. Su, T. Kosa, B. Taheri and T. J. Bunning, Adv. Mater., 2011, 23, 1389-1392. P. J. Ackerman, T. Boyle and I. I. Smalyukh, Nat. Commun., 2017, 8, 673.

P. M. Chaikin and T. C. Lubensky, Principles of condensed matter physics, Cambridge University Press, 1995. J.-S. B. Tai and I. I. Smalyukh, arXiv, 2019, preprint, arXiv: 1911.07829, https://arxiv.org/abs/1911.07829v1. H. R. O. Sohn, P. J. Ackerman, T. J. Boyle, G. H. Sheetah, B. Fornberg and I. I. Smalyukh, Phys. Rev. E, 2018, 97, 052701. R. Swinbank and R. James Purser, Q. J. R. Meteorol. Soc., 2006, 132, 1769-1793.

E. C. Gartland, H. Huang, O. D. Lavrentovich, P. PalffyMuhoray, I. I. Smalyukh, T. Kosa and B. Taheri, J. Comput. Theor. Nanosci., 2010, 7, 709-725. T. Lee, R. P. Trivedi and I. I. Smalyukh, Opt. Lett., 2010, 35, 3447-3449.

P. Ribière and P. Oswald, J. Phys., 1990, 51, 1703-1720.

P. Oswald, J. Baudry and S. Pirkl, Phys. Rep., 2000, 337, 6796.

G. Poy and S. Žumer, Soft Matter, 2019, 15, 3659-3670. H. R. O. Sohn, C. D. Liu and I. I. Smalyukh, Nat. Commun., 2019, 10, 4744.
Express, 2010, 18, 27658-27669.

P. É. E. Cladis and M. Kléman, Mol. Cryst. Liq. Cryst., 1972, 16, 1-20.

L. Gil, J. Phys. II, 1995, 5, 1819-1833.

J. Baudry, S. Pirkl and P. Oswald, Phys. Rev. E, 1998, 57, 3038-3049.

J.-S. B. Tai and I. I. Smalyukh, Opt. Lett., 2018, 43, 51585161.

D. Foster, C. Kind, P. J. Ackerman, J.-S. B. Tai, M. R. Dennis and I. I. Smalyukh, Nat. Phys., 2019, 15, 655-659.

C. Blanc, N. Zuodar, I. Lelidis, M. Kléman and J. L. Martin, Phys. Rev. E, 2004, 69, 011705.

O. Trushkevych, P. J. Ackerman, W. A. Crossland and I. I. Smalyukh, Appl. Phys. Lett., 2010, 97, 201906.

P. Oswald, Europhys. Lett., 2014, 107, 26003.

F. Tu and D. Lee, Langmuir, 2012, 28, 9944-9952.

C. Loussert, S. lamsaard, N. Katsonis and E. Brasselet, Adv. Mater., 2014, 26, 4242-4246.

T. Orlova, S. J. Aßhoff, T. Yamaguchi, N. Katsonis and E. Brasselet, Nat. Commun., 2015, 6, 7603.

A. Varanytsia and L.-C. Chien, Opt. Lett., 2015, 40, 43924395.

H. R. O. Sohn, C. D. Liu, R. Voinescu, Z. Chen and I. I. Smalyukh, arXiv, 2019, preprint, arXiv: 1911.04640, https://arxiv.org/abs/1911.04640v1.

B. Gollapelli and J. Vallamkondu, Curr. Appl. Phys., 2019, 19, 1399-1403. 